\title{
Control Plane Solutions for Sliceable Bandwidth Transceiver Configuration in Flexi-Grid Optical Networks
}

\author{
Ricardo Martínez(1), Filippo Cugini(2), Ramon Casellas(1), Francesco Paolucci(2), Ricard Vilalta(1), Piero \\ Castodi(3), Raul Muñoz(1) \\ (1) Centre Tecnològic de Telecomunicacions de Catalunya (CTTC), Castelldefels (Spain) \\ (2) CNIT, Pisa (Italy) \\ (3) Scuola Superiore Sant'Anna, Pisa (Italy)
}

\section{Abstract}

In Elastic Optical Networks (EONs), the establishment of flexi-grid optical connections requires configuring both bandwidth variable transceivers (BVTs) and bandwidth variable cross-connects (OXC). Such configuration has to be performed through proper control plane architecture and extensions, accounting for the client signal data rate, the required modulation format, the adopted forwarding error correction (FEC) mechanisms, the number of carriers, and the required spectrum resources.

Nowadays, two candidate control plane architectures with similar functions (like endpoint and node addressing, automatic topology discovery, network abstraction, path computation and connection provisioning) can be adopted: (1) the distributed Generalized Multi Protocol Label Switching (GMPLS) - with optional Path Computation Element (PCE) and instantiation/modification - and (2) a control plane based on Software Defined Networking (SDN) with a logically centralized controller and an open protocol such as OpenFlow.

In this work, by relying on the data modelling of 1Tb/s sliceable BVTs (SBVTs), we aim at designing and providing feasible control plane enhancements for both GMPLS/PCE and SDN/OpenFlow architectures to automatically configure endpoint SBVTs while dynamically establishing end-to-end connections in EONs. Such extensions being successfully validated in experimental scenarios are qualitatively compared to ease eventual EON operator selection.

\section{Introduction}

The recent deployment of the flexi-grid technology together with the introduction of coherent detection strategies for high rate optical transmissions have enabled the successful implementation of single-carrier $100 \mathrm{~Gb} / \mathrm{s}$ transponder i.e., Bandwidth Variable Transponder (BVT). Such transmission signal requires frequency slots (FSs) occupying $37.5 \mathrm{GHz}$ of the flexi-grid spectral resources [1]. The advantages of BVTs is to generate optical signals supporting a number of modulation formats, forwarding error correction (FEC) codes and bit-rates that are dynamically selected according to the transported client signals and network conditions (e.g., transmission distance, physical impairments, etc.) [2]. The optical signal being created uses the same FS (defined by the pair of central frequency parameter $n$ and slot width parameter $m$ [3]) on each link between the ingress and egress nodes. This is referred to as spectrum continuity constraint. For each traversed network node, its bandwidth-variable optical cross-connect (BV-OXC) is configured based on the selected FS (i.e., $n$ and $m$ ).

To further improve the overall spectral resource efficiency, flexibility and reconfigurability of EONs, a new generation of transponders, called Sliceable Bandwidth Variable Transponders (SBVTs), is expected to be deployed in the near future [4]. SBVTs support generating multiple flows (subcarriers) each operated at e.g. 100 or $200 \mathrm{Gbit} / \mathrm{s}$ bit rates enabling for instance the creation of super-channel optical connections achieving transmission rates of $1 \mathrm{~Tb} / \mathrm{s}$.

High flexibility in SBVT will be enabled by the capability to configure each subcarrier with different transmission parameters such as the modulation format, the FEC, etc.), to be adapted according to the requested optical reach. Moreover, the subcarriers will have the possibility to be either independently routed 
Within super-channels, no guard bands among subcarriers are required. Instead, guard bands are usually required between independent channels to account for the non-zero roll-off function of the traversed filters, applied by the bandwidth-variable wavelength selective switches (BV-WSS) implemented in the optical BVOXC.

The appealing flexibility benefits brought by SBVTs in EONs also impose important challenges to automatically setting up end-to-end flex-grid connections. The aim of this work is to detail feasible EON control plane enhancements enabling the explicit control of both transit BV-OXCs and reconfigurable SBVT parameters (e.g., modulation format, bit rate, FEC, etc.) at the endpoints. By doing so, the flexibility and benefits in terms of spectrum efficiency provided by EONs are fully leveraged. Hence the protocol enhancements, evaluation and standardization of a control plane for the new generation 1Tbit/s SBVT is addressed. To this end, any control protocol could be used to steer the configuration of the SBVT parameters. However, herein we consider the two most widely adopted network control plane solutions: the traditional GMPLS/PCE control plane technology and the Software Defined Networking (SDN) approach using the OpenFlow protocol. For both solutions, the proposed SBVT-oriented control extensions are specifically designed to support multi-vendor SBVT interoperability, thanks to the definition of a common data information model.

A qualitative comparison between both distributed and centralized solutions for effectively controlling endpoint SBVTs are provided to enable identifying pros and cons associated to each control plane option.

\section{Sliceable Bandwidth Variable Transponder (SBVT)}

This section describes the considered SBVT architecture along with the definition of all the key parameters to be configured when creating either a single optical flow or a super-channel connection (two or more subcarriers). These key parameters represent a generic SBVT data modelling that is then used for the control protocol implementation in either GMPLS/PCE or SDN context.

\section{a. Architecture}

The SBVT, also called Multi-Flow Optical Transponder, enables the client tributary traffic to be transported in the network over a number $C$ of optical subcarriers, whose transmission parameters are configured according to the required optical reach. For example $1 \mathrm{~Tb} / \mathrm{s}$ SBVT can be implemented with $C=10$ subcarriers operating at $100 \mathrm{~Gb} / \mathrm{s}$ Polarization Multiplexed Quadrature Phase-Shift Keying (PM-QPSK) or with $C=5$ subcarriers, each transmitted at $200 \mathrm{~Gb} / \mathrm{s}$ Polarization Multiplexed 16-Quadrature Amplitude Modulation (PM-16QAM).

The architecture of a reference SBVT is reported in Fig. 1 [4], [5]. It includes the following modules:

- Tributary interface. The interface electronically processes the client tributaries. This way, the content provided by the IP layer can be suitably transmitted at the photonic layer. The client traffic is flexibly adapted, according to a predefined granularity (e.g., $100 \mathrm{~Gb} / \mathrm{s}$ ) to $C$ optical flows. The adaptation is typically performed in the framework of the Optical Transport Hierarchy (OTH) within the Optical Transport Network (OTN), as detailed in [6]. The interface also implements key functionalities for FEC mechanisms and operation, administration and maintenance (OA\&M).

- Flow distributor. It is an electronic switching matrix enabling each OTN flow provided by the Tributary interface to be routed towards any subcarrier. The flow distributor is an optional component providing additional flexibility in provisioning and restoration operations.

- Subcarrier generation. Consists of either in an array of $C$ independent laser sources or in a single multi-wavelength source [5]. At the transmitter side, it is used for carrier generation. At the receiver side, it is used as local oscillator.

- Multi-flow module. It consists of $N$ subcarrier modules where each flow is modulated/detected on the related optical subcarrier. At the transmitter side, digital-to-analogue conversion (DAC) is typically exploited to configure multi-level signals for high order modulation formats (e.g., 16QAM). Shaping filters are also included to improve transmission performance. At the receiver side, coherent detection is exploited. In particular, high-speed analog-to-digital converters (ADCs) are exploited. 
- $\quad$ Multiplexer/Coupler. All sub-carriers are optically coupled/multiplexed into a single add/drop port of the BV-OXC. The BV-OXC typically consists on a "broadcast or switch and select" architecture implemented with BV-WSS [7].

\section{b. Super-Channel and SBVT data model}

For the above considered SBVT architecture, a number of key parameters are required to be configured for generating a super-channel connection and for each individual subcarrier. In this sense, Fig. 2.a depicts a (class) diagram via Unified Modeling Language (UML) determining the data model to be used when setting up a super-channel (formed by one or more subcarriers, see example in Fig. 2.b) and configuring each involved subcarrier.

The parameters and attributes related to the super-channel creation include:

- $\quad$ Number of active subcarriers (a non-zero positive integer). In the case of fully loaded SBVTs, all C subcarriers are activated to carry the tributary traffic at full rate. Optionally, under networking conditions where the full rate is not required, only a subset $c(\leq C)$ of the available subcarriers may be activated.

- $\quad$ Super-channel frequency slot (freq_slot). The frequency/optical spectrum range allocated to the super-channel connection. As stated above a FS is completely defined by its nominal central frequency (NCF) using the parameter $n$ and its slot width (parameter $m$ ). In the data model, we use the object label to specifically determine the FS. Label format (IETF RFC 7699) is defined by the grid parameter (i.e., ITU-T Flex [3] in EONs), the channel spacing (CS) specifying the spacing between adjacent channels (e.g., $6.25 \mathrm{GHz}$ ), the identifier used to distinguish different lasers within the same node when they can transmit the same wavelength, the $n$ parameter representing an offset with respect to an anchor frequency (193.1 THz),], and the parameter $m$ defined as a multiple of a given slot width granularity (i.e., $12.5 \mathrm{GHz}$ ).

The super-channel FS information is used to configure the BV-WSS within all traversed BV-OXCs along the entire connection.

- Spatial Path (path). It specifies the route/path in terms of the sequence of nodes and links from the ingress to the egress of the connection. This determines the BV-OXCs that need to be configured to accommodate the super-channel with its specified FS.

The capabilities and features related to each subcarrier forming the super-channel (described in Fig. 2.a) include:

- $\quad$ Subcarrier frequency slot. The spectrum utilization of each subcarrier is identified by the label object (i.e., grid, CS, identifier and the NCF defined by the integers $n$ and $m$ ). To achieve ultra-high spectral efficiency, a debate is ongoing to relax the $6.25 \mathrm{GHz}$ granularity to $\mathrm{CS}$ of $1 \mathrm{GHz}$ or even to any frequency value of the spectrum. The subcarrier NCF $(n)$ is used to configure the laser source at the transmitter and the local oscillator at the receiver. The subcarrier slot width $(m)$ can be used to determine the proper filter shaping configuration within each subcarrier module.

- Baud rate (baud_rate). The baud rate (in symbol/s) of each subcarrier is configured according to the available electronic processing capabilities of the transponder, in such a way that, considering the applied modulation format and FEC/Coding, the generated gross rate is able to guarantee the requested net bit rate. Typically, a restricted set of candidate values is considered. The baud rate configuration is applied to the subcarrier module.

- Modulation format (mod_format). Pool of supported modulation formats. Examples include PMQPSK and PM-16QAM. To be applied within the Subcarrier module.

- $\quad F E C / c o d i n g$ (fec_coding). At the transmitter side, to guarantee adequate robustness, specifically designed redundancy (i.e., overhead) is applied to the net bit rate. FEC is typically applied within the OTN operations at the Tributary interface. Examples of FEC are: reed-solomon, hamming code, $\mathrm{BCH}$, etc.

- $\quad$ Central Frequency Granularity (CFG): it indicates the multiple of CS between supported adjacent NCFs. Fig. 2.b depicts the viable NCFs for a subcarrier according to the supported CFG.

- Slot Width Granularity (SWG): it determines the slot width granularity (in multiple of $2^{*} \mathrm{CS}$ ) between two consecutive FS generated by the same subcarrier. Fig. 2.b depicts some examples about the feasible FS width depending on the subcarrier SWG. 
- Minimum Slot Width (min_width): it indicates for a subcarrier the minimal slot width can be generated.

- Maximum Slot Width (max_width): it indicates for each subcarrier the maximum slot width that can be generated. Observe that specifying both min_width and max_width the range of feasible FSs to be generated by subcarrier ide strictly defined.

To successfully set up super-channel connections, the aforementioned parameters need to be adequately considered and incorporated in the adopted control plane approach as detailed in the next section.

\section{Control Plane Solutions for Flexi-Grid Optical Networks}

A control plane aims at fulfilling the requirements of fast and automatic end-to-end provisioning and rerouting of flexi-grid connections, covering common functions like addressing, automatic topology discovery, network abstraction, path computation, connection provisioning and recovery.

The control plane functions can, on a first approach, be distributed or centralized. Either way, they need to be extended to address the new requirements associated to the aforementioned optical technologies: adequate robustness and efficient flexible spectrum allocation of co-routed connections (i.e., super-channel establishment and configuration). The selection of a centralized or distributed control plane is conditioned by aspects such as flexibility and extensibility, availability, already installed deployments, actual network size and scalability. In section $\mathrm{V}$, we qualitatively provide some discussions addressing those aspects with respect to the selected control plane approach.

\section{a. GMPLS/PCE Distributed Control Plane}

A set of cooperating entities (controllers) execute the control plane functions in a distributed manner. Each controller governing a network node (e.g., BV-OXC) disseminates the topological elements that are directly under its control via a routing protocol (e.g., Open Shortest Path First, OSPF). The routing dissemination enables each control plane entity having a unified view of the network topology i.e., node/link connectivity and network resource availability. Such information allows computing end-to-end QoS-enabled services at either the connection ingress node or via a centralized dedicated element referred to as Path Computation Element (PCE). Afterwards, the connection is set up by relying on a signaling protocol (Resource Reservation Protocol, RSVP) along the nodes involved in the computed path. The reference architecture is defined by the ITU-T ASON and relies on the GMPLS set of protocols defined by the IETF. A data communication network, based on IP control channels allows the exchange of control messages between GMPLS controllers. Each GMPLS controller manages the state of all the connections (i.e., LSPs) originated, terminated or passing-through a node, and maintains its own network state information (topology and resources), collected in a local Traffic Engineering Database (TED). Figure 3.a shows the distributed GMPLS-based control plane architecture along with the exchanged control protocol messages used to discover the network topology (OSPF-TE), computing end-to-end paths via PCE protocol (PCEP) and establishing the flexi-grid LSPS (RSVP-TE).

\section{b. SDN/OpenFlow Control Plane}

A single entity (controller) is responsible for the control plane functions, commonly using open and standard protocols, such as the OpenFlow protocol (OF/OFP). The SDN controller performs path computation and service provisioning, configuring the forwarding and switching behavior of the nodes. A centralized control plane provides a method for programmatic control of network resources and simplification of control plane process. By deploying the control plane intelligence in the controller, resources allocated in hardware nodes for control plane functions can be reduced. Such solutions may involve deploying hardware (computational and storage) which is orders of magnitude more powerful than individual controllers. Figure 3.b depicts the (logically) centralized SDN controller being responsible for setting up the connections directly configuring (via OFP FLOW MOD messages) the involved BV-OXC nodes. That is, the switching (WSS) of BV-OXCs are modified according to the FS of the flexi-grid connection being established.

\section{Control Plane Enhancements in Support for SBVT}

Regardless of the adopted control plane solution, the automatic and dynamic configuration of flexi-grid networks must take into account the functionalities, capabilities and restrictions imposed by devices such as the (S)BVTs. This is done to attain the most efficient use of spectrum resources when serving end-to-end 
connections. In this section, we detail the required extensions in terms of protocols and processes considering the data modeling described above (i.e., data model of section II.b) for the effective control of SBVTs in the context of both GMPLS and SDN/OFP protocol extensions.

\section{a. GMPLS Protocol Extensions}

205

206

207

208

The GMPLS OSPF-TE protocol provides to each controller the network topology (i.e., graph) and TE link and node attributes such as the link metric, the link NCF availability, the switching node restrictions, etc. This information is gathered into repositories (TED) at each GMPLS controller and at the centralized PCE. By doing so, the PCE is able to compute end-to-end paths taking into account the specifics of the optical layer.

The characteristics and capabilities of SBVTs are thus required when computing end-to-end flexi-grid optical paths, especially to compose super-channels connections. With this aim, both GMPLS OSPF-TE and RSVPTE protocols are extended [8]. In general, whenever a link or node attribute is modified (e.g., resources are de/allocated for connection setting up/tear down) an OSPF-TE Link State (LS) Update message is flooded within the network domain updating controllers' TED. The link and node attributes carried into the LS Update message are formatted via Type Length Value (TLVs) structure. Particularly, to flood SBVT TE attributes the existing GMPLS Port Label Restriction (PLR) sub-TLV is extended as depicted in Fig. 4.b. Such SBVT information along with the topology graph and the NCF link availability constitute the necessary input to devise routing and spectrum assignment (RSA) algorithms at PCE and enabling on-line computation of feasible path connections, thus improving the overall spectrum efficiency [8].

The carried SBVT attributes in the GMPLS PLR sub-TLV are:

- $\quad$ TxSubTrnsp and RxSubTrnsp specifying the number of total/equipped subcarriers for Tx and Rx direction on the interface, respectively.

- $\quad$ AvailTxSubTrnsp and AvailRxSubTrnsp specifying the number of available subcarriers (i.e., not used) for Tx and Rx directions, respectively.

- $\quad$ Aggregated NCF status for both Tx and Rx directions on the interface between the SBVT device and the BV-OXC (see Fig. 1). To this end, a standard OSPF-TE Label TLV is used where a bitmap coding allows identifying by each bit the status of a supported NCF: 1 means available and 0 occupied.

o Num labels specifies the supported number of NCFs (which determines the size of the bitmap coding).

- Grid, CS, Identifier, $n$ and $m$ form the standard lambda label for EONs (cfr. IETF RFC 7699) as aforementioned. Using the bitmap coding, the parameter $n$ specifies the lowest NCF (e.g., anchor frequency of $193.1 \mathrm{THz}$ ) which is used as the reference wavelength to determine the frequency of each supported NCF

With the above information at the time of computing and selecting the resources (i.e., optical spectrum and SBVT subcarriers) for a new connection, the RSA algorithm is aware whether sufficient unused subcarriers are available at the endpoints' SBVT and whether the optical spectrum continuity and contiguity can be ensured [8]. For the sake of completeness, in EONs without wavelength conversion, the RSMA need to deal with two constraints: i) the spectrum continuity constraint, i.e. the same unused optical spectrum portion should be allocated on each link of the path; ii) the spectrum contiguity constraint, i.e., the NCFs occupied by the pool of subcarriers forming the super-channel need to be spectrally contiguous. Finally, observe that other relevant SBVT information (described in the data model of Fig. 2.a) such as the list of supported modulation formats and FEC coding could be easily added into the extended PLR sub-TLV contents.

Once the route is computed by the RSA algorithm at the PCE, RSVP-TE conveys the necessary information to allow resource reservation and configuration of the optical sub-systems (i.e., SBVTs and BV-OXCs). To do that, the path is passed as an explicit route object (ERO) to the RSVP-TE protocol. The ERO contents or sub-objects are formed by an ordered sequence of nodes and links to be traversed (i.e., unnumbered interface IDs) dealing with the spatial_path parameter in the data model of Fig. 2,a, followed by the computed FS (i.e., $n$ and $m$ parameters) carried within the ERO Label subobject. This label information corresponds to the freq_slot parameter of the super-channel shown in Fig. 2.a.

To handle the SBVT configuration at the connection endpoints, the RSVP-TE protocol is extended carrying the so-termed Explicit Transponder Control (ETC) subobject into the Path message [8]. The ETC subobject 
depicted in Fig. 4b is formed by a variable list of Transponder (subcarrier) TLVs. Each Transponder TLV contains four sub-TLVs enabling the configuration per subcarrier basis:

- $\quad$ Sub-Transponder (subcarrier) Id locally identifies a subcarrier within the SBVT.

- $\quad$ Subcarrier-FS specifies the FS $(n$ and $m$ ) to be allocated by the respective subcarrier.

- Modulation Format configures the selected subcarrier modulation format (e.g., QPSK, 8-QAM, etc.).

- $\quad$ FEC allowing its configuration.

Observe that the above subcarrier configuration attributes are directly bound to the subcarrier specific parameters described into the data model depicted in Fig. 2.

\section{b. OpenFlow Protocol Extensions}

OFP version 1.3 supports optical flows provisioning and control. However, flexi-grid is not supported yet and a number of significant parameters and attributes are missing to properly configure SBVTs. Initial proposals for extensions specifically suitable for flexi-grid optical networks and SBVT have been presented [9],[10].

The OFP FLOW MOD message sent by the SDN Controller to the OF agent is responsible for the configuration of the flow entries in each node. The selection of the SBVTs and the BV-OXC to accommodate the connection is performed by the path computation process used by the SDN controller. Once the end-toend path is computed, to support configuring SBVTs the FLOW MOD has to be extended. First, the flexigrid FS (e.g., grid type, CS, $n$ and $m$ parameters) is required to be configured at each BV-OXC (i.e, BV-WSS filters of the selected output port of the node). Then, the configuration of the selected SBVT at the connection endpoints indicates the card transmission parameters encompassing the following attributes (see Fig. 5):

- $\quad$ Single/multi carrier optical channel.

- Number of subcarriers forming the super-channel,

- Specification of the subcarriers including baud rate (and thus supported bit rate).

- NCF (i.e., parameter $n$ ).

- Subcarrier width (parameter $m$ ),

- Modulation format.

- $\quad$ FEC, code type and rate.

For the sake of completeness, it is worth mentioning that such attributes follow the data model presented in Fig. 2.a at the time of setting up super-channel connections and configuring their respective set of subcarriers.

When a single co-routed media channel is computed, a single FLOW MOD per node is forwarded. Whereas, when sliceability is considered, the configuration of different media channels originating from the same SBVT is provided with different FLOW MOD messages (possibly bundled, if originating from combined computation), each one with different configurations of the BV-OXC output ports and BV-WSS filters. Therefore, such extensions fully enable the sliceable functionality.

Specific extensions enable the implementations of sliceability comprising programmable and asymmetric multiwavelength (PAMW) signal generation [11]. In particular, the laser type, laser id and tone id attributes identify the laser source type physical laser and the laser instance utilized for that flow. BV-OXC and SBVT configurations are acknowledged by means of asynchronous or synchronous mechanisms (e.g., using extended PORT STATUS messages or novel FLOW ACK messages).

The extensions imply that detailed SBVT information (e.g., node architecture, type and number of installed transponders, node/card capabilities) are available at the controller performing advanced path computation including spectrum and transmission parameters assignment. Node/SBVT information can be provided by means of either discovery procedures or enhanced controller-agent OpenFlow session handshake describing the OpenFlow switch architecture and available capabilities.

Table I.a summarizes the key features for each of the above control plane approaches to support automatic SBVT configuration in EONs. For the sake of clarification, these features include the key control plane functions such as the service provisioning, the required routing information and the path computation.

\section{Conducted Experimental Validations of the SBVT-controlled Approaches and Qualitive Comparison}


In [9], the authors conducted an experimental validation and performance evaluation of the GMPLS SBVToriented protocol extensions (described in section IV.a) to dynamically serve flexi-grid LSPS. The tests were performed at the control plane level within the CTTC ADRENALINE testbed. A 14-nodes Spanish EON topology was used where each link supports $128 \mathrm{NCFs}$ (with a channels spacing of $6.25 \mathrm{GHz}$ ). Every connection/LSP request to be served besides specifying the source and the destination endpoints (equipped with SBVTs) demands a specific bit rate (ranging from 100 to $500 \mathrm{~Gb} / \mathrm{s}$ ). The collected TED information (i.e., topology, link spectrum resource and SBVTs attributes) flooded by the OSPF-TE protocol, is used as input for a RSMA algorithm executed at the PCE. If a feasible connection is computed, the output is formed by: the spatial path (ERO) i.e. nodes and links to be traversed, the connection FS ( $n$ and $m$ ), and the number and ids of the subcarriers (constituting the super-channel LSP) to be activated along with their modulation format and FEC configuration.

The attained results in [8] allow reflecting the feasibility of the proposed GMPLS routing and signaling extensions in support of SBVTs configuration upon dynamic flexi-grid traffic along with supporting the automatic establishment of super-channels connections. The RSMA algorithm (in average) takes $10 \mathrm{~ms}$ for the path computation while the signaling process requires (in average) $40 \mathrm{~ms}$ to set up the end-to-end connection, not including hardware latencies.

The OpenFlow extensions reported in Sec. IV-B have been employed in a real SDN data/control plane experimental testbed at CNIT labs. The EON data plane includes SBVT prototypes with advanced functionalities generating configurable PM-QPSK-modulated super-channels at up to $1 \mathrm{~Tb} / \mathrm{s}$ exploiting Time Frequency Packing (TFP) technique and coherent detection; commercially available BV-WSSs with configurable filter of $1 \mathrm{GHz}$ granularity; fixed-length optical links (optical amplifiers, fiber spans) and configurable optical recirculating loop to setup different optical reach distances. Such devices are controlled and dynamically configured by OpenFlow-enabled agents called FlexSwitch, collocated at each BV-OXC. OpenFlow sessions are established among each FlexSwitch and the southbound interface of the central OpenFlow controller called FlexController [9], capable of lightpath setup, tear down and dynamic adaptation. In particular, adaptation options include path rerouting, elastic operations (e.g., additional subcarrier activation due to bandwidth increase request), hitless spectrum shifting and code rate change based on the TFP technique [12]. The FlexController stores a TED and a flow database, which are kept updated by information retrieved by FLOW ACK messages in the case of controller-driven actions, and by PORT STATUS messages in the case of asynchronous events (e.g., failure, QoT degradations). The TED is enriched with information related to node architecture, functionalities and available modules. The controller implements impairment-aware path computation also accounting for the SBVT capabilities at source and destination (i.e., available/configurable physical parameters). Different path computation and adaptation algorithms are utilized, mainly based on least congested spectrum K-shortest path subject to spectrum continuity constraint and QoT validation, and may include multiple (either in parallel or sequential fashion) actions output [13].

Results on OpenFlow-based control plane testbed show that path computation at the controller (including spectrum and TFP-based transponder transmission parameter assignment) is performed within $5 \mathrm{~ms}$ for provisioning [12] and within 100ms for multipath restoration [9]. Node configuration, exploiting OpenFlow messages, is performed in a parallel fashion and therefore, is practically independent from the number of involved nodes. The configuration requires a time contribution within $5 \mathrm{~ms}$ for OpenFlow control plane (i.e., including message exchange, flow entries processing and TED update) and a variable time ranging from $40 \mathrm{~ms}$ up to $1.6 \mathrm{~s}$ for hardware configuration (e.g., actual filter re-shaping enforcement of BV-OXC) [12].

Concerning computation related to advanced SBVT features, a significant example refers to PAMW sources generating multiple sliceable subcarriers. In this case, the OCH SPEC information carried by each FLOW MOD to source and destination SBVTs specifies the selected laser source identifier and multiple (incremental) laser tone identifiers. The extended FLOW MOD message (Fig. 5) captured at the FlexController southbound interface during PAMW experimental validation in which three sliceable lightpath setup requests and configurations have been performed. In this specific case, three lightpaths are activated, generated by the same physical laser source and routed along two different paths (port 1 for flows 1 and 2 , and port 2 for flow 3) through proper BV-OXC configuration. The capture shows the complete flow configuration of 3 lightpaths including request, configuration messages, acknowledgments and final reply enabling flow traffic utilization. 
The above summarized experiments and experiences conducted by the authors allow validating the proposed protocol extensions in each control plane approach to support the objective of automatically configuring SBVTs when setting up flexi-gird connections. In addition, the experimental tests provide also detailed insights when comparing both control plane approaches. In Table I.b. both control plane approaches are qualitatively compared in terms of pros and cons using a number of performance parameters such as the technology matureness, the scalability, the extensibility complexity, the multi-vendor interoperability issues, the multi-domain support, the recovery, etc. In general, each approach presents its own advantages and disadvantages here outlined aiming at supporting network operators at the time of selecting one option or the other.

\section{Conclusions}

This work focuses on the recent advances attained in the context of the EU IDEALIST project [14] for the effective control of EON infrastructures taking into account the configuration of SBVTs at the endpoints. In this regard, first a generic data model addressing most of the capabilities and features of SBVTs is provided (e.g., number of subcarriers, modulation formats, FEC, etc.). This is made with the special interest on supporting the appealing flexi-grid SBVT advantage of creating super-channel connections (e.g., $1 \mathrm{~Tb} / \mathrm{s}$ ) as a result of merging a number of subcarriers. The adopted data model represents baseline to define two control plane approaches to enable the automatic control and configuration of SBVTs, in EON. The control plane architectures are the traditional distributed GMPLS and the centralized SDN/OpenFlow. In both cases, we have presented design considerations and the proposed protocol extensions and procedures. To illustrate and validate each of the control plane approaches, two scenarios have been detailed and executed in two experimental testbeds at CTTC and CNIT labs. Furthermore, a qualitative comparison in terms of different technical aspects (e.g., scalability, matureness, multi-vendor interoperability support, etc,) is provided. In light of the obtained results in both experimental activities, we can state the feasibility of the adopted control plane approaches which leaves the network operator the option to select any of them for controlling their EON network.

\section{Acknowledgments}

This paper was supported by the European Community's Seventh Framework Programme FP7/2007-2013 through the IDEALIST project (grant agreement $\mathrm{n}$. 317999) and the Spanish Ministry of Economy and Competitiveness (MINECO) through the project DESTELLO (TEC2015-69256-R).

\section{References}

[1] A. Lord, P. Wright, and A. Mitra. "Core networks in the flexgrid era." IEEE/OSA J. of Lightw.Tech vol.33, no. .5, pp. 1126-1135, 2015:

[2] Y. Sone, et al., "Bandwidth squeezed restoration in spectrum sliced elastic optical path networks (SLICE)" IEEE/OSA J. Opt. Commun. Netw., vol. 3, no. 3, March 2011.

[3] "ITU-T Recommendation G.694.1, Spectral grids for WDM applications: DWDM frequency grid."

[4] N. Sambo et al., "Next generation sliceable bandwidth variable transponder," IEEE Communications Magazine, 2015.

[5] N. Sambo, et. al., "Sliceable transponder architecture including multiwavelength source" IEEE/OSA J. J. Opt. Commun. Netw., vol. 6, no. 7, pp. 590-600, July 2014.

[6] "Interfaces for the optical transport network (OTN)," ITU-T Recommendation G.709, Dec. 2009.

[7] M. Jinno, et. al., "Multiflow optical transponder for efficient multilayer optical networking", IEEE Commun. Mag., vol. 48, no. 8, August 2010.

[8] R. Martínez, et. al., "GMPLS/PCE-Controlled Multi-Flow Optical Transponders in Elastic Optical Networks", IEEE/OSA J. J. Opt. Commun. Netw., vol. 7, no. 11, pp. B71-B80, November 2015.

[9] F. Paolucci, A. Castro, F. Cugini, L. Velasco, and P. Castoldi, "Multipath restoration and bitrate squeezing in SDN-based elastic optical networks [invited]," Springer Photonic Netw. Commun., vol. 28, pp. 45-57, 2014. 
[10] L. Liu, et. al., "Demonstration of a dynamic transparent WSON employing flexible 404 transmitter/receiver controlled by an OpenFlow/stateless PCE integrated control plane," in Proc. of Optical 405 Fiber Communication Conference and Exposition (OFC) and The National Fiber Optic Engineers 406 Conference (NFOEC), Anaheim, California, USA, March 2013.

407 [11] N. Sambo, et. al., "First demonstration of SDN-controlled SBVT based on multi-wavelength source 408 with programmable and asymmetric channel spacing," in Proc. of Optical Communication (ECOC), 2014 409 European Conference on, Sept 2014, pp. 1-3.

410 [12] N. Sambo, et. al., "Programmable transponder, code and differentiated filter configuration in 411 elastic optical networks," IEEE/OSA J. of Lightw.Tech., vol. 32, no. 11, pp. 2079-2086, June 2014.

412 [13] F. Paolucci, et. al., "Active pce demonstration performing elastic operations and hitless defragmentation 413 in flexible grid optical networks," Springer Photonic Netw. Commun., Svol. 29, no. 1, pp. 57-66, 2015.

$414 \quad$ [14] EC FP7 IP project "Industry-Driven and Adaptive Lambda Infrastructure for Service and Transport 415 Networks (IDEALIST”, http://www.ict-idealist.eu/

\section{Biographies}

Ricardo Martínez (SM'14) graduated and $\mathrm{PhD}$ in telecommunications engineering by the UPCBarcelonaTech university in 2002 and 2007, respectively. He has been actively involved in several publicfunded (national and EU) R\&D as well as industrial technology transfer projects. Since 2013, he is Senior Researcher of the Communication Networks Division (CND) at CTTC. His research interests include control and network management, protocols and traffic engineering mechanisms for packet and optical transport networks within aggregation and core segments.

Filippo Cugini is Head of Research at CNIT, Pisa, Italy. His main research interests include theoretical and experimental studies in the field of optical communications and networking. He is co-author of twelve patents and more than 200 international publications.

Ramon Casellas (SM'12) graduated in telecommunications in 1999 by the UPC and ENST. He worked as an undergraduate researcher at FT R\&D and BT Labs, completed a Ph.D. in 2002 and worked as an associate professor (ENST) until joining the CTTC in 2006. He is a senior research associate, involved in R\&D and technology transfer projects. His research interests include network control GMPLS/PCE and SDN/NFV. He has co-authored over 150 papers and contributes to IETF.

Francesco Paolucci received the Laurea degree in Telecommunications Engineering in 2002 from

433 University of Pisa, Italy and Ph.D. degree in 2009 from Scuola Superiore Sant'Anna, Pisa, Italy. In 2008, he was granted with a research Merit Scholarship at INRS, Montreal, Canada. Currently, he is Assistant Professor at Scuola Superiore Sant'Anna. His main research interests are within transport network control plane (GMPLS, PCE and SDN). He is co-author of four international patents and more than 100 international publications.

Ricard Vilalta (Girona, 1983) has a telecommunications engineering degree (2007) and Ph.D. degree (2013), at UPC, Spain. Since 2010, he is researcher at CTTC, in the Communication Networks Division. He is currently a Research Associate at Open Networking Foundation. His research is focused on SDN/NFV, Network Virtualization and Network Orchestration. He has been involved in international, EU, national and industrial research projects, and published more than 100 journals, conference papers and invited talks.

Piero Castoldi (SM) has been Associate Professor at Scuola Superiore Sant'Anna, Pisa, Italy since 2001 444 where he leads the "Networks and Services" area. He had led roles in the following EU projects: BONE, STRONGEST, IDEALIST, OFELIA, FED4FIRE, 5GEx. His research interests cover telecommunication network architectures: optical network control, traffic engineering and reliability, and data center architectures. He is author of more than 300 publications in international journals and conference proceedings and has also filed a total of 20 patents. 
and System Department. Since 2000, he has participated in several public-funded R\&D (EC and Spanish) and technology transfer projects. He has led several Spanish R\&D projects and the EU-Japan STRAUSS

453 project STRAUSS. Research interests include control and management architectures, protocols and traffic engineering algorithms for future transport networks. 


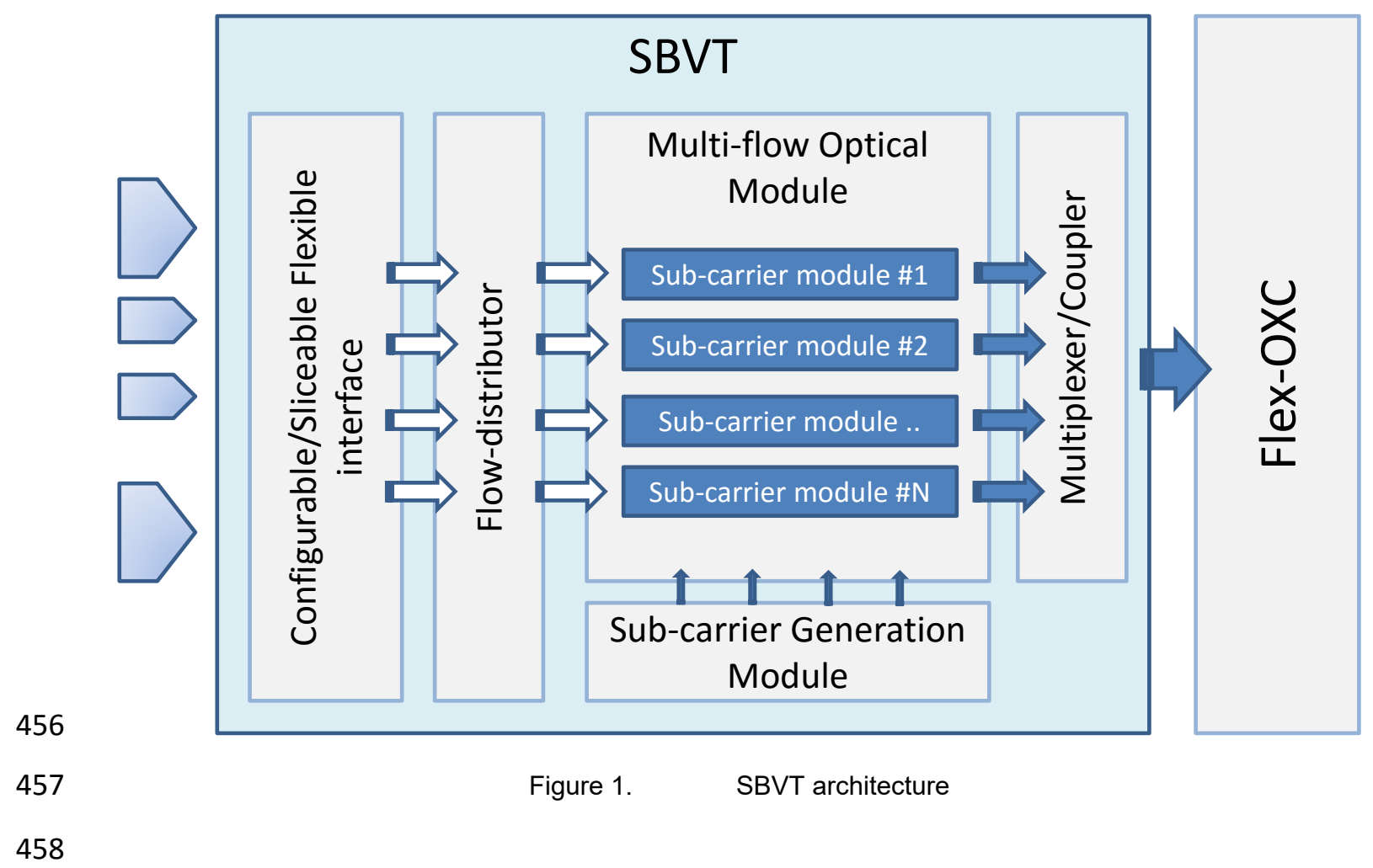



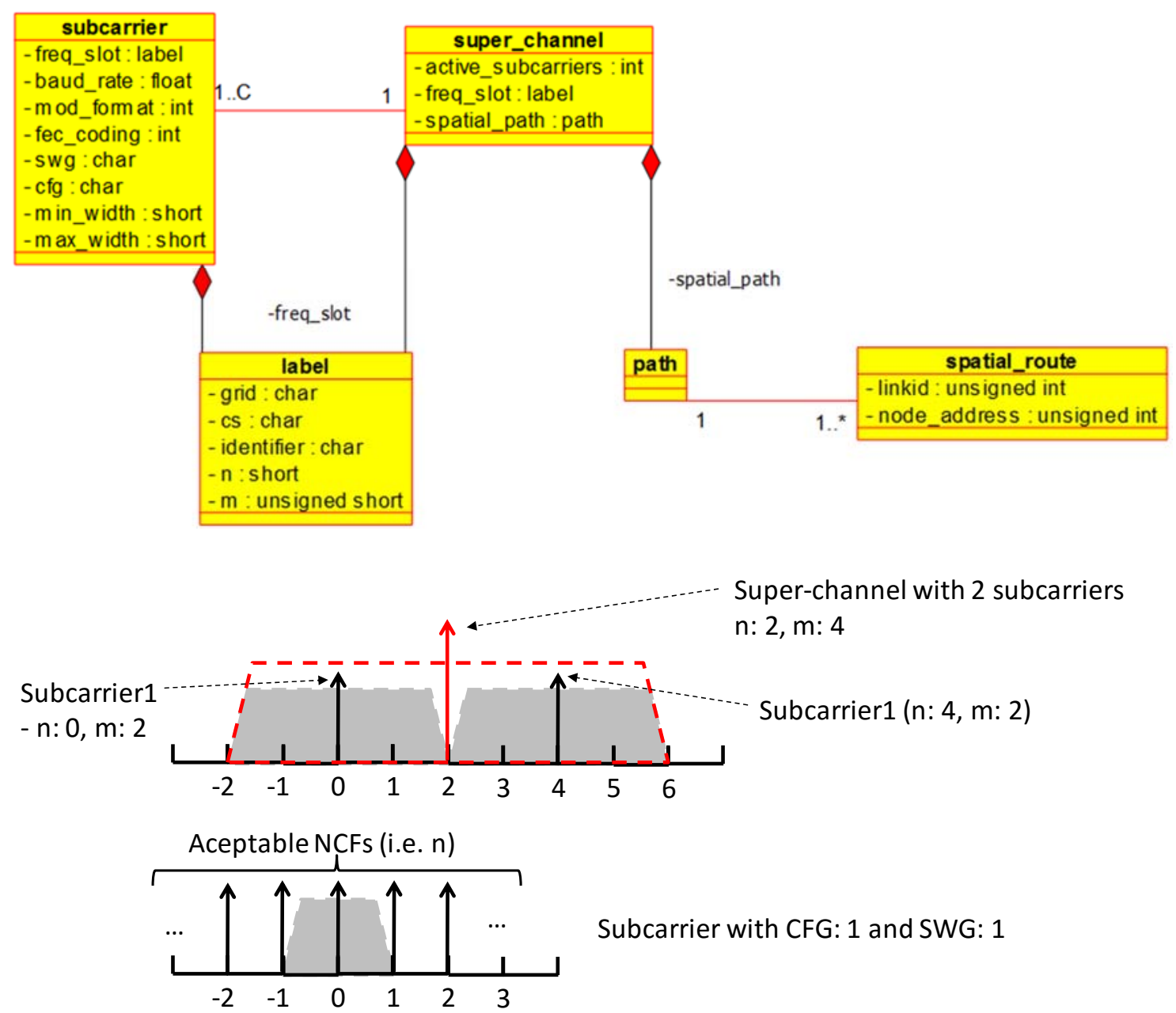

Subcarrier with CFG: 1 and SWG: 1

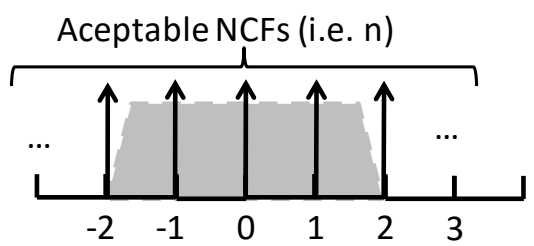

Subcarrier with CFG: 1 and SWG: 2

Aceptable NCFs (i.e. $n$ )

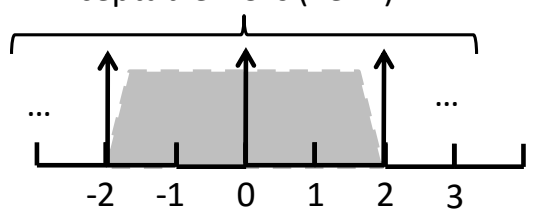

Subcarrier with CFG: 2 and SWG: 2

(a) UML for the super-channel and SBVT creation and configuration; (b) Examples illustrating a super-channel formed by two subcarriers and candidate subcarrier configuration according to 

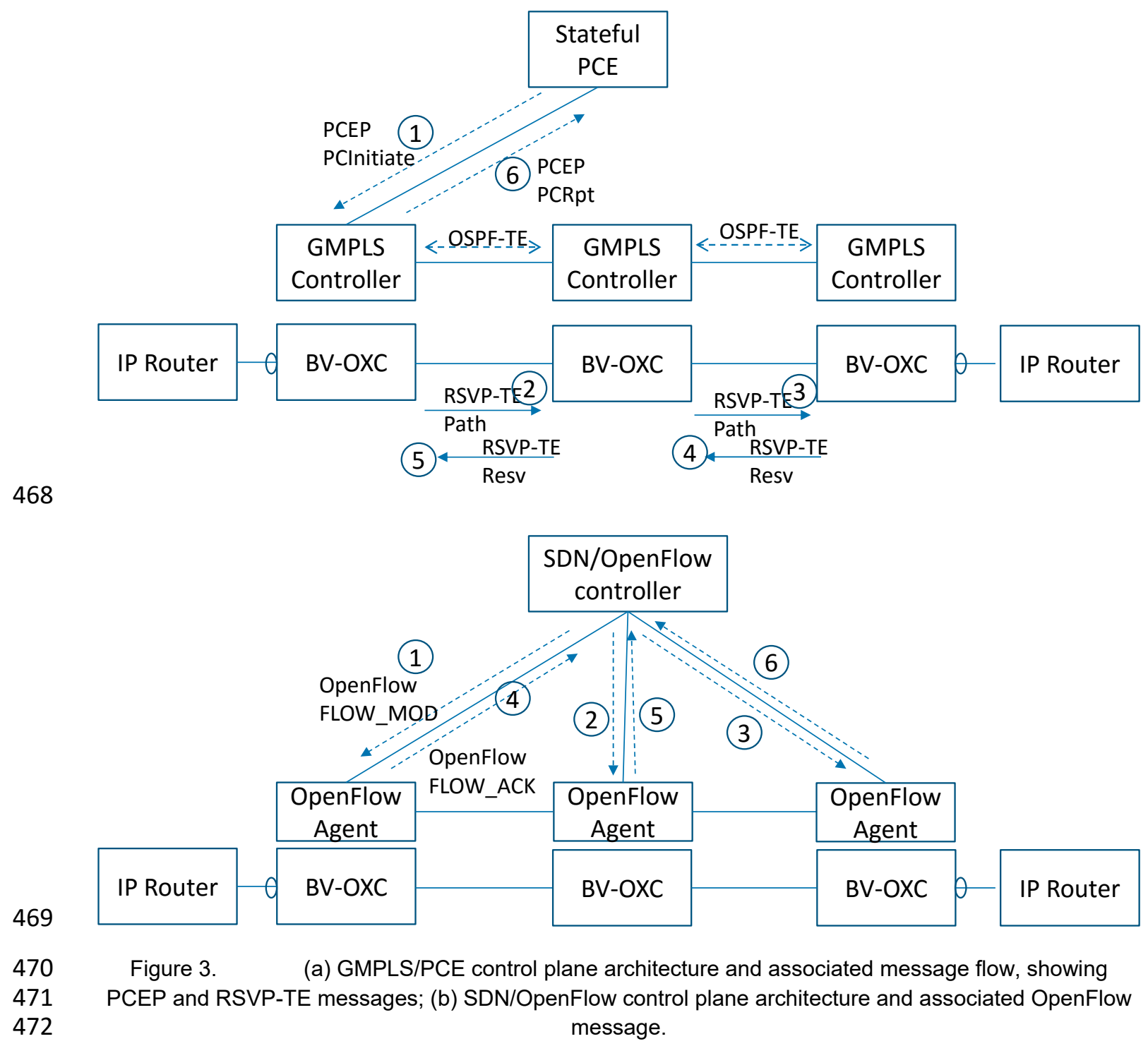


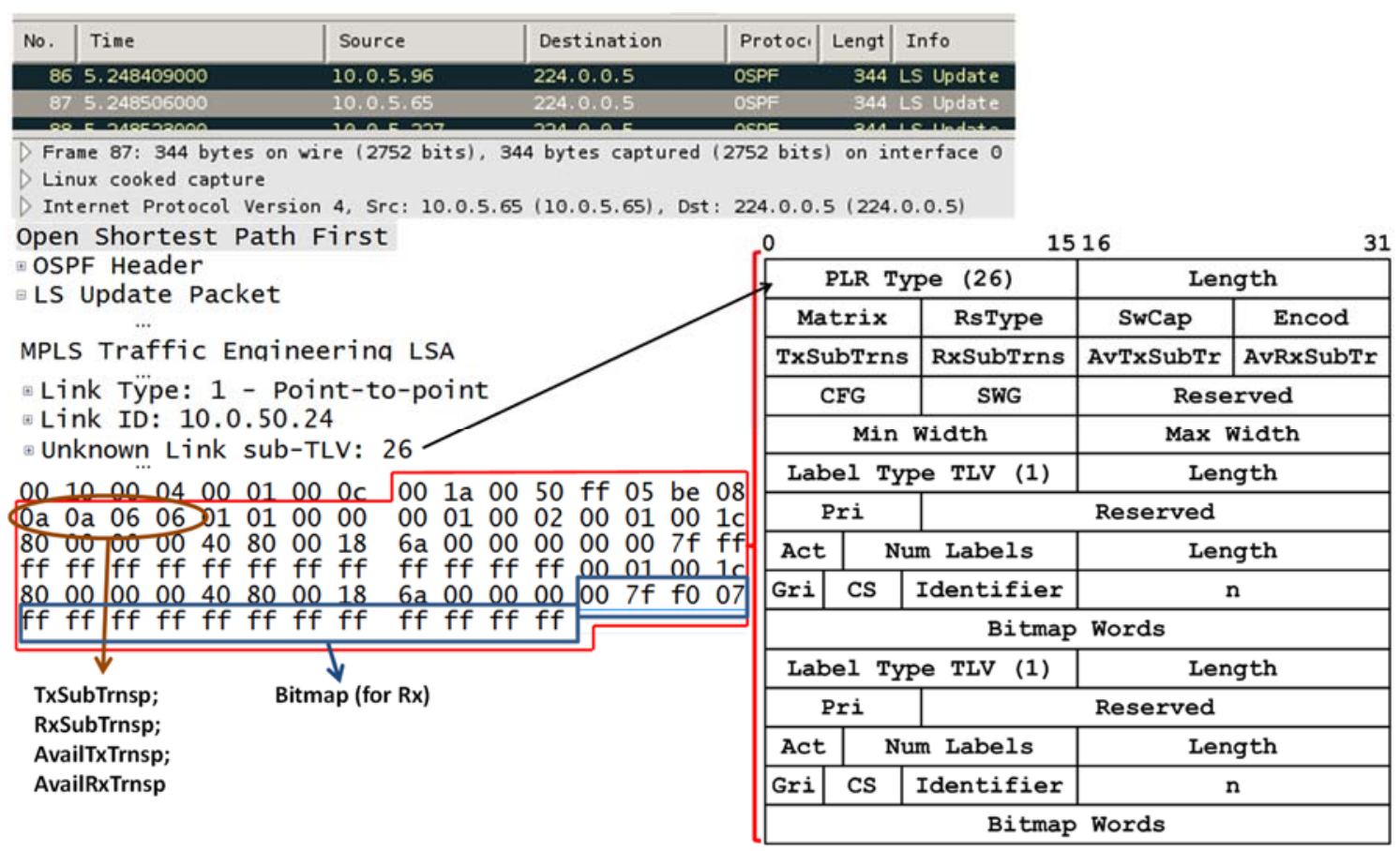

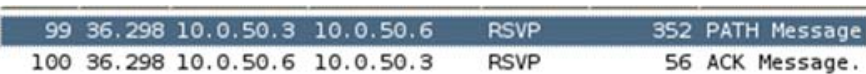

$>$ Frame 99: 352 bytes on wire ( 2816 bits), 352 bytes captured ( 281

Linux cooked capture

Internet Protocol Version 4, Src: $10.0 .50 .3(10.0 .50 .3)$, Dst: 16

$\checkmark$ Resource ReserVation Protocol (RSVP): PATH Message. SESSION:

EXPLICIT ROUTE: Unnum 10.0.50.6/20, Labe1 1778384916 .

Length: 128

object class: EXPLICIT ROUTE object (20)

C-type: 1

Unnumbered Interface-ID - 10.0.50.6, 20, Strict

- Labe1 Subobiect - 1778384916. Strict

* Unknown subobject: 10

$\begin{array}{lllllllllllllllll}00 & 04 & 00 & 00 & 0 a & 64 & 00 & 01 & 00 & 01 & 00 & 30 & 13 & 8 d & 00 & 08\end{array}$

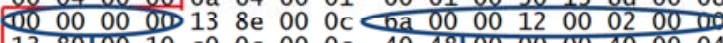

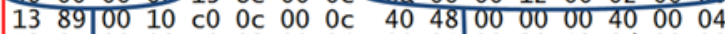
\begin{tabular}{ll|llllllll|lllllll}
13 & $8 \mathrm{a}$ & 00 & 08 & $\mathrm{c} 0$ & 03 & 00 & 04 & 00 & 01 & 00 & 30 & 13 & $8 \mathrm{~d}$ & 00 & 08
\end{tabular}

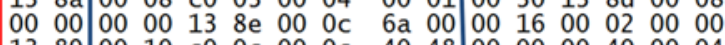

\begin{tabular}{ll|llllllll|llllll}
13 & 89 & 00 & 10 & $c 0$ & $0 c$ & 00 & $0 c$ & 40 & 48 & 00 & 00 & 00 & 40 & 00 & 04
\end{tabular}

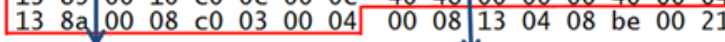

Sub-Trnsp Id

Label: $\mathrm{n}: 12 ; \mathrm{m}: 2$

\begin{tabular}{|c|c|c|c|c|c|c|}
\hline \multicolumn{2}{|c|}{-Trnsp Id } & \multicolumn{2}{|c|}{ Label: $\mathrm{n}: 12 ; \mathrm{m}: 2$} & \multicolumn{2}{|c|}{ Type (5005) } & Length \\
\hline \multicolumn{2}{|r|}{ Type (5001) } & Tonath & \multicolumn{4}{|c|}{ Transponder Id } \\
\hline si & Modulation ID & Length & \multicolumn{3}{|c|}{ Type (5006) } & $T$ ond \\
\hline \multicolumn{3}{|c|}{ Symbol Rate } & Gri & cs & Identifier & $\mathrm{n}$ \\
\hline \multicolumn{2}{|r|}{ Num Carriers } & Bits/Symbol & \multicolumn{3}{|r|}{$m$} & Reserved \\
\hline
\end{tabular}

\begin{tabular}{|c|c|}
\hline Type (5002) & Length \\
\hline FEC ID & Length \\
\hline
\end{tabular}

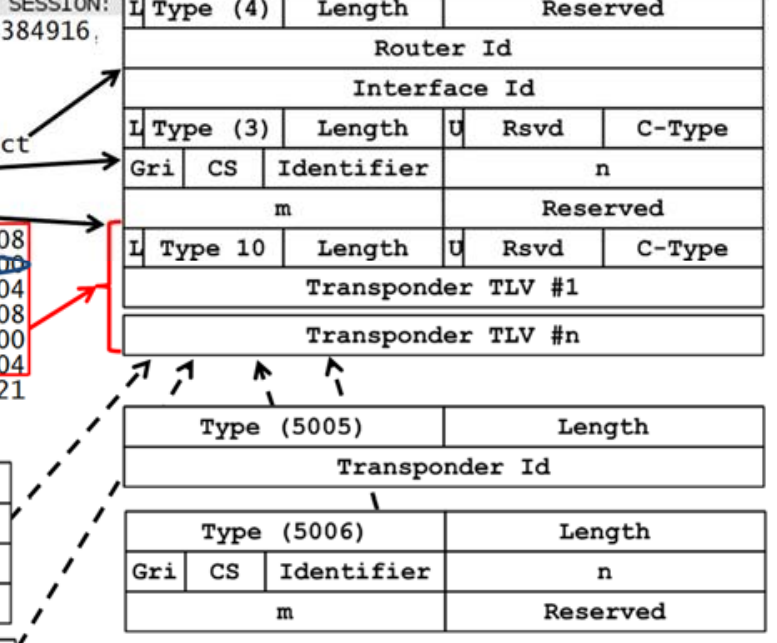

Figure 4.

(a) GMPLS OSPF-TE PLR sub-TLV extensions in support of SBVTs; (b) GMPLS RSVPTE ERO Transponder subobject extension in support of SBVTs 


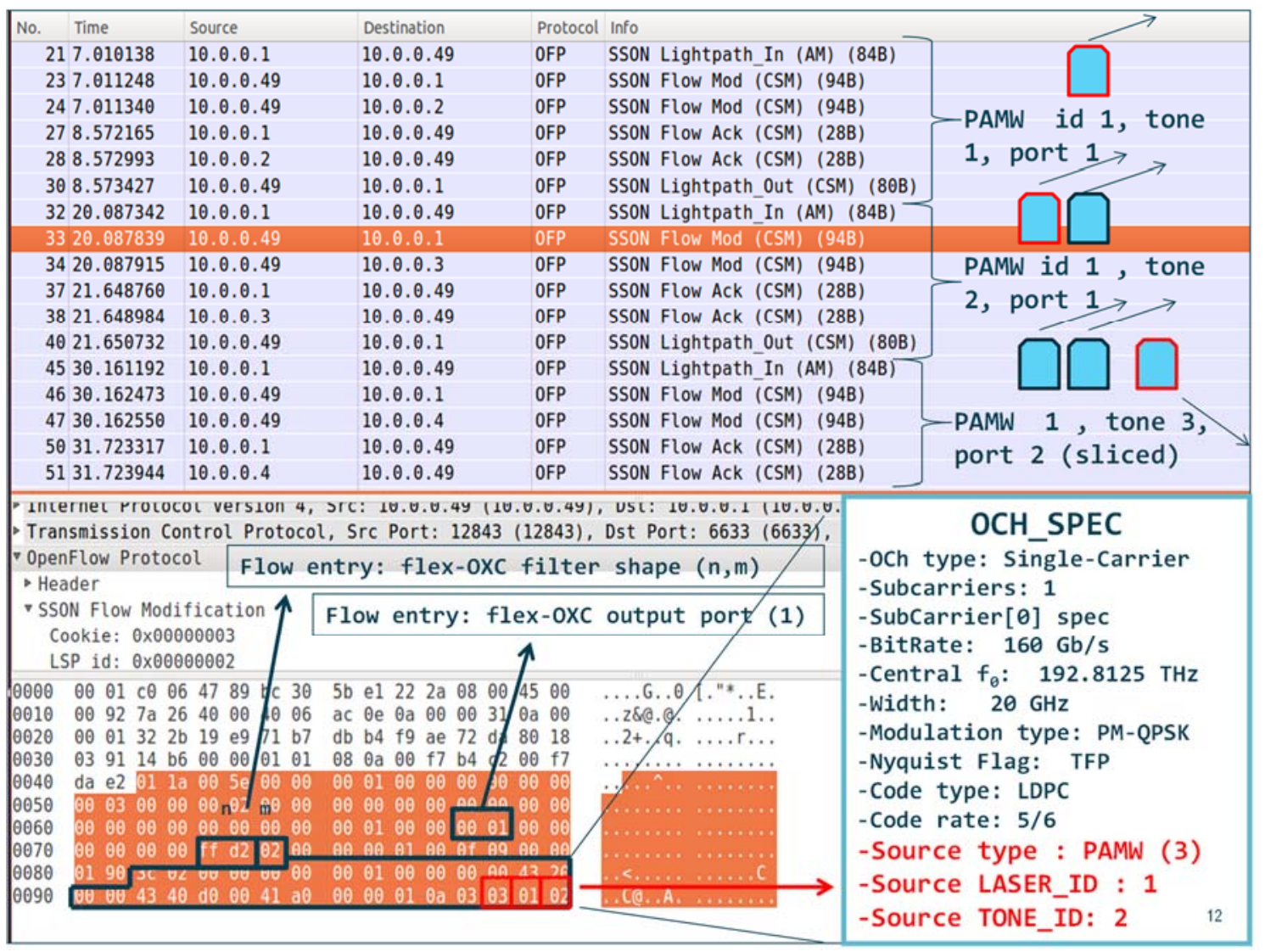

Figure 5. Capture of extended OpenFlow messages for SBVT lightpath setup. 


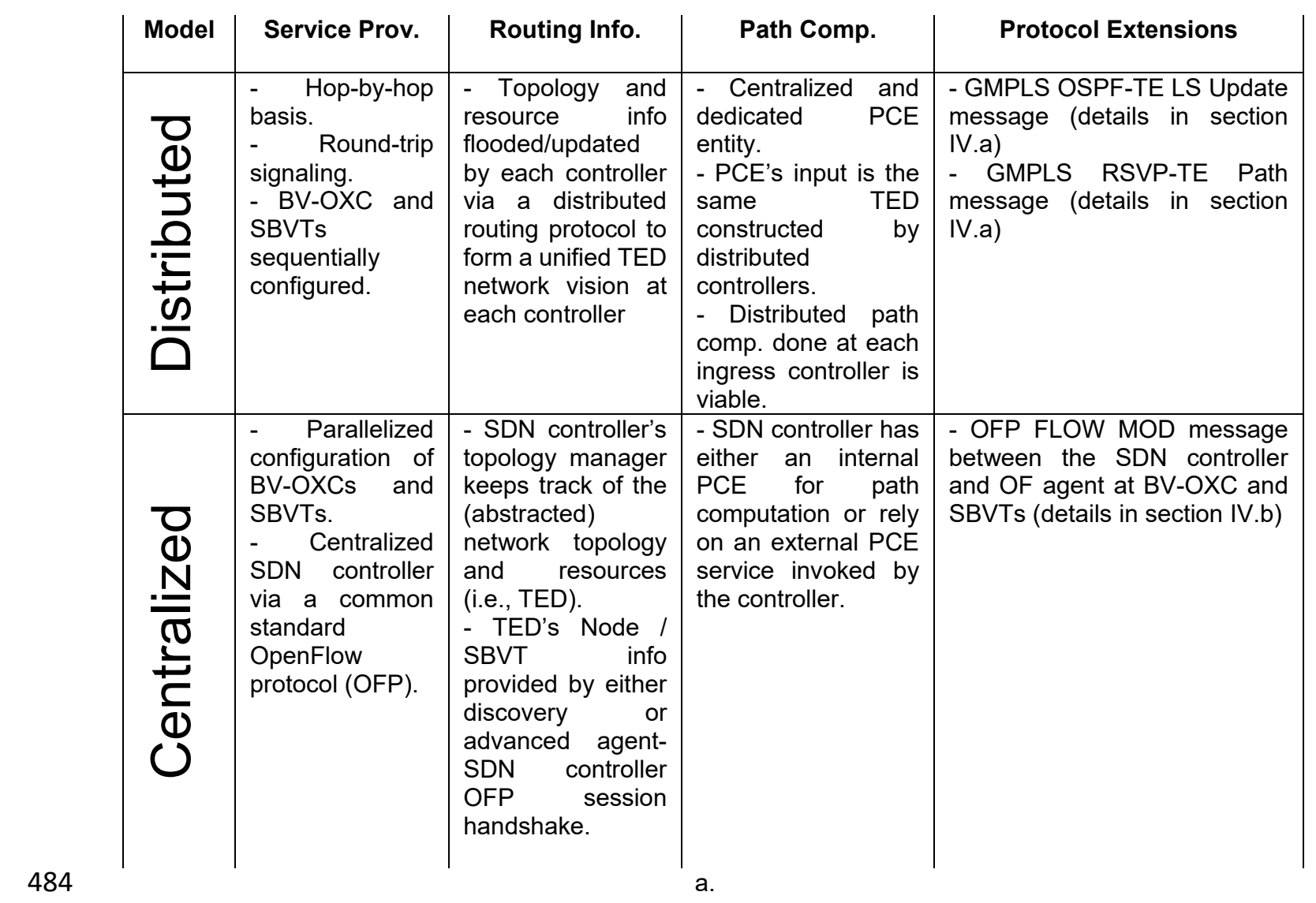

\begin{tabular}{|c|c|c|}
\hline \multirow{2}{*}{ Model } & \multirow[b]{2}{*}{ Pros } & \\
\hline & & Cons. \\
\hline & $\begin{array}{l}\text { - Mature for both packet and transport networks. } \\
\text { - Available commercial deployments. } \\
\text { - Simple extensibility for SBVT attributes. } \\
\text { - Effective multi-domain support e.g., via peering } \\
\text { PCEs (network abstraction). } \\
\text { - Good performance under moderate/high } \\
\text { dynamic traffic environments. } \\
\text { - Good scalability with respect to network size. } \\
\text { - Effective and rapid response if service failures } \\
\text { occur. }\end{array}$ & $\begin{array}{l}\text { - High complexity (different protocols and } \\
\text { processes). } \\
\text { - Multi-vendor interoperability issues. } \\
\text { - Under very high dynamic traffic scenario, } \\
\text { control overhead (e.g., updating SBVT } \\
\text { capabilities) may be cumbersome. } \\
\text { - TED convergence time may lead to cause } \\
\text { out-of-dated path decisions under network } \\
\text { failures affecting a number of services. }\end{array}$ \\
\hline & $\begin{array}{l}\text { - Mature for packet networks. } \\
\text { - Effective unified control plane. } \\
\text { - Lower control plane (protocol) complexity. } \\
\text { - Use of standard and open protocol (avoid } \\
\text { vendor proprietary extensions). } \\
\text { - Effective multi-vendor interoperability. } \\
\text { - Enhanced programmability and flexibility (e.g., } \\
\text { virtualization). } \\
\text { - No out-of-dated information impacting } \\
\text { performance. } \\
\text { - Support of multi-domain adopting hierarchical } \\
\text { SDN control. } \\
\text { - Adding SBVT support in OFP and assuming } \\
\text { high dynamic traffic scenario the resulting control } \\
\text { overhead is not a big issue. }\end{array}$ & $\begin{array}{l}\text { - Not mature for transport networks (some } \\
\text { prototypes). } \\
\text { - Scalability issues when network size } \\
\text { increases due to centralized control } \\
\text { (bottleneck effect). } \\
\text { - Latency issues if congestion in SDN } \\
\text { controller under highly dynamic traffic or } \\
\text { network failures. } \\
\text { - Robustness problems if centralized } \\
\text { controller fails. }\end{array}$ \\
\hline
\end{tabular}

b.

Table I. a. Main features of distributed and centralized control plane solutions to support automatic SBVTs 